\title{
ZOMBIES OU DIPLOMATES : ONTOLOGIES DES SPORTS EXTRÊMES
}

\author{
GAUTHIER DIERICKX
}

\begin{abstract}
The objective of the paper consists in the examination of the ambiguous relationship between ecology and extreme sports in order to highlight both the opportunities and impasses the latter represent for contemporary ecological issues. Indeed, extreme sports are generally only perceived as the embodiment of what Ghassan Hage calls "generalised domestication". This concept represents a way of living and constituting a surrounding world and the others in which everything is deemed worthwhile only insofar as a certain value can be extracted from them. However, I shall argue that they could as well propose ontological alternatives to this generalised domestication by creating a world underlain by mutual relationships and "diplomacy" with Nature and others.
\end{abstract}

\section{Introduction}

Au cours d'un voyage en Bolivie en juillet 2018, je fis l'ascension de l'Huyana Potosi et descendis en VTT El camino de la Muerte. Deux activités relevant de ce que d'aucun.e.s nomment les "sports extrêmes ", c'est-à-dire, des activités pour lesquelles une simple erreur ou un banal accident peuvent occasionner la mort du ou de la participant.e concerné.e ${ }^{1}$.

Deux activités nécessitant normalement des heures d'entrainement, un savoir-faire bien rodé, et surtout une connaissance pointue du milieu environnant, que j'ai enchainées en seulement septante-deux heures. Sur le moment, je ne me suis même pas posé la question de ce que cet enchaînement pouvait bien dire sur

1 Brymer Eric, Gray Tonia, « Dancing with Nature: Rhythm and Harmony in Extreme Sport Participation », in Journal of Adventure Education and Outdoor Learning, 9/2, 2009, p. 136.

https://doi.org/10.14712/24646504.2020.4

(C) 2020 The Author. This is an open-access article distributed under the terms of the Creative Commons Attribution License (http://creativecommons.org/licenses/by/4.0). 


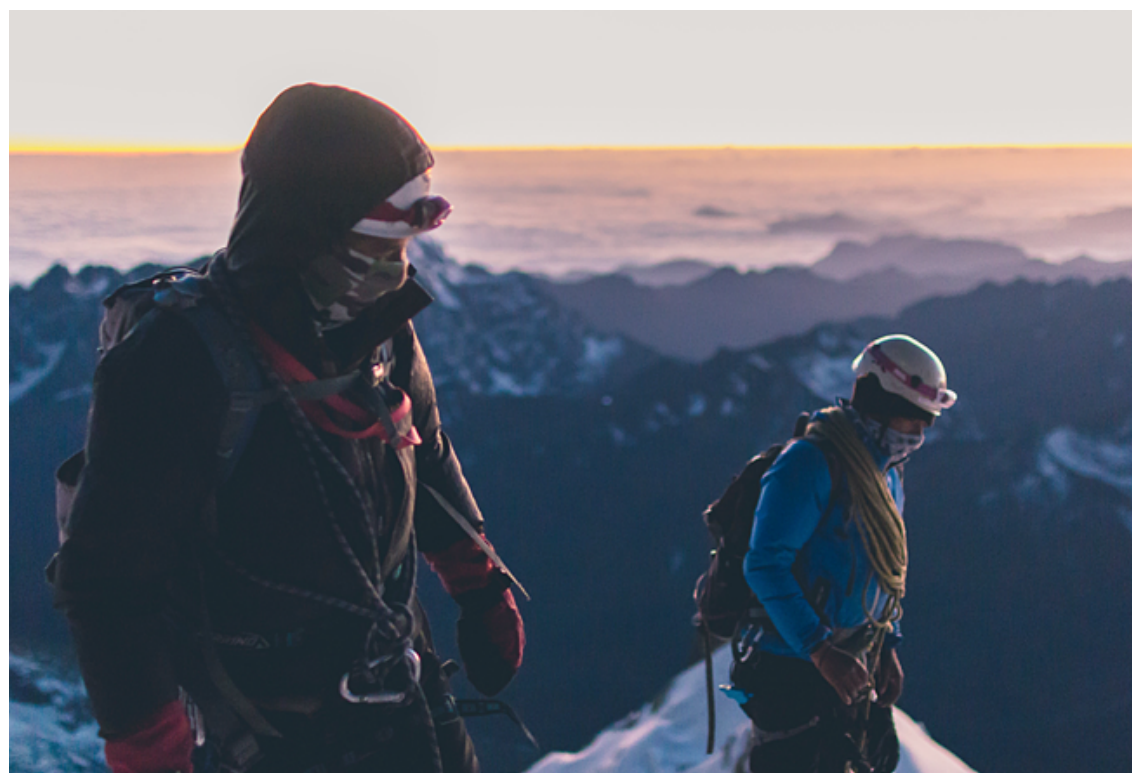

Au sommet de l'Huayna Potosi ${ }^{2}$, un mastodonte de neige et de glace, à plus de 6080 mètres d'altitude, au cœur de la Cordière des Andes.

ma relation au monde et aux altérités. Je voulais saisir tout ce que ce voyage avait à m'offrir, pouvoir exhiber fièrement ces exploits, montrer à tout un.e chacun.e de quelle trempe j'étais fait. Alors quoi de plus normal que de considérer le monde comme un immense terrain de jeu, dont la valeur dépend uniquement de la manière dont il peut renforcer mon sentiment de puissance et témoigner de mon caractère exceptionnel ?

Je n'avais jamais fait d'alpinisme, et guère plus de vélo de descente. Je ne connaissais rien à rien. Je n'avais jamais utilisé un piolet de ma vie, encore moins des crampons. Je n'avais jamais rencontré la montagne, tenté de négocier avec elle. Et il en allait de même pour ces virages serrés vertigineux, où une simple faute d'inattention avait déjà coûté la vie à une quinzaine de cyclistes, très souvent aussi inexpérimenté.e.s que moi. Mais malgré cela, je me suis lancé à l'aventure, à la conquête de ces deux monuments. Sans préparation aucune, simplement

2 Photo : Coen Tomas @tomyyco, Aurore au sommet du mont Huayna Potosi. Une expérience aussi belle que glaciale, 2018. Reproduite avec l'aimable autorisation de l'artiste. 


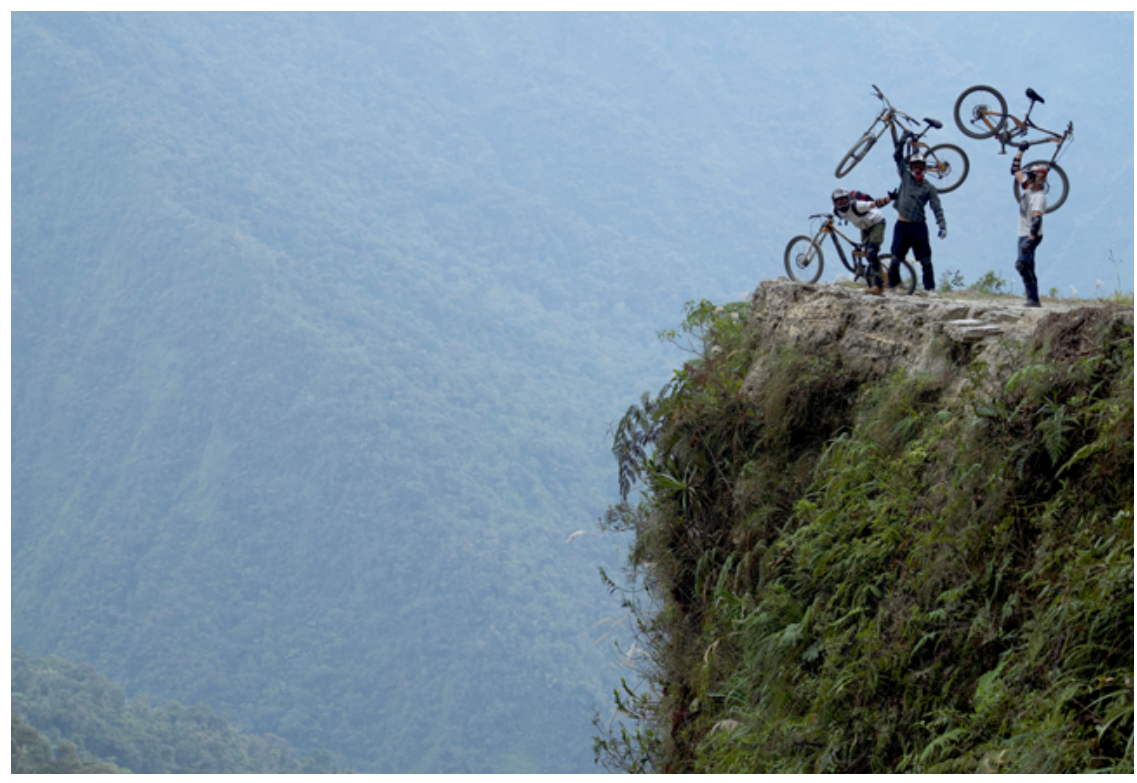

El Camino de la Muerte ${ }^{3}$, ou la route de la Mort. Voie vertigineuse entre les villes de La Paz et de Coroico. Descente escarpée de soixante-quatre kilomètres le long de falaises abruptes, sur un étroit chemin de terre sinueux, creusé de nids-de-poule et de crevasses.

accompagné de guides locaux chevronnés, chargés de négocier à ma place avec les éléments. Je les écoutais attentivement, les suivais avec déférence, peut-être conscient malgré tout que, pour eux, cette montagne ou cette piste était autre chose que cette insouciante arène du dépassement de soi, que ce simple miroir dans lequel je contemplais fièrement mon courage et ma détermination.

C’est précisément « l'ambiguïté ontologique » de ces pratiques extrêmes que j'aimerais analyser dans cet article, en confrontant deux des « fantasmes de viabilité $»^{4}$ qui les animent, afin d'interroger à la fois les possibilités et les impasses qu'elles représentent pour les enjeux écologiques contemporains. Ces fantasmes

Photo : Bugnon Sébastien, Tuni taita, 2019. Reproduite avec l'aimable autorisation de l'artiste.

4 Par «fantasme de viabilité », Ghassan Hage désigne la « création d'un espace qui fait sens [et] par lequel, en même temps, on donne du sens à la vie, comme une vie valant la peine d'être vécue ". Hage Ghassan, Le loup et le musulman. Le racisme est-il une menace écologique?, Marseille, Éditions Wildproject, 2017, p. 76. 
s'inscrivent ainsi dans une certaine conception ontologique ${ }^{5}$ en vertu de laquelle le, ou mieux, les mondes sont littéralement engendrés par nos propres corps. Chaque corps, par les affects qui le singularisent, par ses puissances et ses faiblesses respectives, constitue un monde singulier. ${ }^{6}$ Nos « manières d'être $»^{7}$, nos genres de vie sont ainsi créateurs de mondes, à travers la relation que nous entretenons avec ceux-ci. Perception et signification du monde y sont indiscernables, tout en demeurant distinctes, à l'instar de l'objet et de son reflet dans le miroir ${ }^{8}$. Ainsi, comme l'explique Vinciane Despret : " Percevoir, c'est accorder des significations. N'est perçu que ce qui a une signification, comme ne reçoit de significations que ce qui peut être perçu, et qui importe pour l'organisme. " ${ }^{9} C^{\prime}$ est dans un même mouvement que nous percevons le monde et lui donnons un certain sens. Nous l'organisons en fonction du sens que nous donnons à la vie, de ce que nous considérons comme " une vie valant la peine d'être vécue " ${ }^{10}$, à laquelle correspond une manière d'être spécifique, un mode de vie singulier. Ainsi, il y a certainement tout autant de différences entre le cheval de labour et le cheval de course ${ }^{11}$ qu' entre les deux éthos de sports extrêmes que je propose d'analyser ici. Bien qu'homonymes, ces pratiques renvoient en fait à des ontologies radicalement différentes, constituées par des manières d'affecter et d'être affectées distinctes, par des « mode[s] de relation au monde qui, au cours du processus de mise en relation, crée[nt] le monde même auquel il[s] se met[tent] en relation $»^{12}$.

En effet, si certaines manières de vivre et de réaliser ces pratiques se situent dans la continuité du fantasme de viabilité consubstantiel à la modernité occidentale, qualifié de " domestication généralisée » ${ }^{13}$ par Ghassan Hage, tout en l'actualisant à l'ère du capitalisme financier, d'autres sont au contraire tout à fait à même de constituer une puissante alternative ontologique - et écologique - à ce fantasme,

5 On retrouve cette conception notamment chez : Deleuze Gilles, Spinoza. Philosophie pratique, Paris, Editions de Minuit, 1981 ; Von Uexkül Jakob, Milieu animal et milieu humain, Rivages, 2010; Viveiros de Castro Eduardo, Métaphysiques cannibales, Paris, PUF, 2009; Despret Vinciane, Que diraient les animaux, si... on leur posait les bonnes questions ?, Paris, La Découverte, 2012.

6 Viveiros de Castro E., Métaphysiques Cannibales, op. cit., p. 39.

7 Ibid., p. 40.

8 Deleuze G., Cinéma 2. L'image-temps, Paris, Editions de Minuit, 1985, pp. 92 et s.

9 Despret V., Que diraient les animaux..., op. cit., p. 222.

10 Hage G., Le loup..., op. cit., p. 76.

11 Selon Gilles Deleuze, « il y a de plus grandes différences entre un cheval de labour ou de trait, et un cheval de course, qu'entre un bœuf et un cheval de labour. C'est parce que le cheval de course et le cheval de labour n'ont pas les mêmes affects ni le même pouvoir d'être affectés ; le cheval de labour a plutôt des affects communs avec le bœuf. » Deleuze G., Spinoza..., op. cit., p. 167.

12 Hage G., Le loup..., op. cit., p. 76.

13 Ibid., p. 75. 
en ce qu' elles témoignent d'une conception bien plus « mutualiste » ou « diplomatique » du monde et des autres.

\section{Les sports extrêmes et la « subjectivité zombie »}

Certaines de ces pratiques extrêmes, loin de transformer radicalement notre rapport constitutif au monde et aux autres, s'inscrivent en fait pleinement dans le sillon tracé par la domestication généralisée. Pour ce fantasme de viabilité caractéristique de la modernité occidentale, « la principale qualité de tout ce qui naît est 'd'exister' pour quelque chose ${ }^{14}$, de produire de la valeur - matérielle, financière, esthétique, symbolique, etc. - pour un sujet domestiquant. ${ }^{15}$ Le monde ainsi engendré est fondamentalement organisé en fonction de l'intérêt personnel de cette subjectivité, des sensations et avantages qu' elle peut en extraire ${ }^{16}$, au détriment des altérités qui y sont soit exploitées, soit exclues, voire exterminées. ${ }^{17}$ Hégémonique, cette conception du monde tend à l'homogénéisation et la mise à l'écart de tout ce qui ne participe pas pleinement aux intérêts du sujet, tout ce qui n'augmente pas, d'une façon ou d'une autre, son sentiment de maitrise et d'exceptionnalité. La vie " valant la peine d'être vécue " y est avant tout une vie à l'abri des effets déstabilisateurs des altérités, réduites au rang de ressources et/ou de menaces.

\subsection{Domestication généralisée - Terre vierge et viol}

Les « lieux sauvages » dans lesquels les sports extrêmes prennent place sont habituellement considérés comme de simples aires de jeu, organisées exclusivement en fonction de la valeur que peuvent en extraire les individus. Pour certain.e.s pratiquant.e.s, il est avant tout question de chercher un spot inconnu, un sommet inexploré, une paroi à la neige immaculée pour violer une nature intacte, la dépuceler, se l'approprier, ne lui accorder de l'importance qu'en tant qu' elle leur appartient, qu'elle porte désormais leur trace. La nature se résume alors pour iels à un simple « outil servant à la réalisation de soi ${ }^{18}$.

\footnotetext{
Ibid., p. 76.

Ibid., p. 80.

Ibid., p. 86.

Idem.

8 Le Breton David, Conduites à risque, Paris, PUF, 2013, p. 254.
} 
En témoigne par exemple ce film promotionnel de l'équipementier français Picture Organic clothing, Zabardast ${ }^{19}$, dans lequel une équipe de riders décident de se lancer dans le pari un peu fou de se rendre au Pakistan pour dévaler une montagne, la Tour Nord Biachearhi, « verticale, jamais skiée " ${ }^{20}$ insiste la voix off du film. Pour eux, c'est sa virginité qui en constitue l'attrait, comme pour mieux y laisser leur trace, mieux se l'approprier. Thomas, à l'origine de l'escapade, rêve de surfer cette paroi depuis qu'il en a découvert l'existence dans un livre emprunté à la bibliothèque. ${ }^{21}$ Et après que les sherpas ont quitté les riders à 4400 mètres d'altitude, il répète inlassablement, en plein milieu de la montagne : "On est tout seul, on est tout seul $»^{22}$, preuve de la pauvreté ontologique ${ }^{23}$ dans lequel son monde est plongé. Celui-ci n'est peuplé que d'autres lui-même. Dans la glace qui l'entoure, Thomas ne voit que son propre reflet.

Il n'est pas anodin que le vocabulaire du viol marque autant leur rapport à la nature. Ces pratiquant.e.s ne cherchent la pureté que pour mieux la souiller. Iels veulent qu' elle soit vierge jusqu'à la nuit de noces, là où elle sera enfin leur. Comment ne pas faire le parallèle ici avec le fameux slogan de la propagande sioniste selon lequel la Palestine serait « une terre sans peuple pour un peuple sans terre » ? Comment ne pas comprendre que ces deux rapports au monde participent d'une même structure ontologique, d'une seule et même conception du monde?

Dans cette optique, ce qui les dérange fondamentalement dans la présence d'autrui au sein de tels " paradis perdus ", ce n'est pas que l'autre se les accapare ou les colonise, mais bien qu' il le fasse avant iels, qu'il occupe déjà le terrain, sapant ainsi les prétentions de leur puissance extractive, déniant leur caractère exceptionnel.

Partant, autrui n'est jamais conçu comme un ennemi, mais uniquement comme un concurrent, un alter ego, cherchant lui aussi à accroître sa puissance. Dans leur monde, il n'y a pas de concurrence ontologique, juste une ontologie concurrentielle. De la sorte, on saisit mieux pourquoi de telles pratiques cadrent si bien avec la logique de marché. Car si ces sports extrêmes représentent aujourd'hui

19 Tanon Jérôme, Zabardast, https://www.youtube.com/watch?v=AkigzUFr3ys\&t=88s (consulté le 27 juin 2019).

20 Ibid., 8 min. 43 s. (consulté le 27 juin 2019).

21 Ibid., 8 min. 40 s. (consulté le 27 juin 2019).

22 Ibid., 14 min. 35 s. - 14 min. 36 s. (consulté le 27 juin 2019).

23 Par "pauvreté ontologique ", je désigne l'impossibilité pour ce pratiquant de se laisser affecter par les altérités, de constituer un monde qui ne serait plus organisé exclusivement en fonction de son intérêt propre, mais qui ferait au contraire la part belle aux affects de ces altérités, aux mondes que celles-ci engendrent à travers leur propre corporalité. 
un marché colossal ${ }^{24}$, c'est qu'ils ont d'abord constitué un monde-en-tant-quemarché, c'est-à-dire un monde qui n'existe que pour son accaparement, son extraction. Tout marché économique implique nécessairement un marché ontologique préalable.

\subsection{La « subjectivité zombie » - capitalisme et cannibalisme}

L'engouement général que suscitent aujourd'hui certaines de ces pratiques au sein de la société occidentale s'inscrit d'ailleurs dans la lignée de la "nouvelle politique de subjectivation ${ }^{25}$ qui émergea au tournant des années 1980 avec le capitalisme " financier ", appelé aussi « culturel » ou « culturel-informel ${ }^{26}$. Contrairement au capitalisme industriel, la plus-value n'y est plus fondamentalement extraite de quelque force de travail, mais bien d'une « force de connaissance et d'invention ${ }^{27}$, produisant en roue libre des identités et des modes de vie formatés, «prêts-à-porter ${ }^{28}$, auxquels sont sommé.e.s de s’identifier les consommateur. trice. $\mathrm{s}^{29}$

L'ego contemporain n'est désormais plus enfermé dans des identités relativement stables, discontinues, mais est contraire une hybridation sans cesse rejouée, illimitée..$^{30} \mathrm{Il}$ est tout à la fois plongeur, grimpeur, voltigeur, etc. Semblable à une vague qui « enroule ce qui autour se déroule et déroule ce autour de quoi elle s'enroule » ${ }^{31}$, il enchaîne les identités à un rythme effréné, les collectionne comme des trophées de chasse. L'ego est désormais une activité frénétique d'extraction d'expériences et de sensations, dont la valeur dépend du capital d'identités et de compétences accumulées ainsi que de sa «flexibilité » ${ }^{32}$, c'est-à-dire, sa capacité à se sentir immédiatement partout chez lui, sans avoir à négocier avec les altérités qui l'environnent ${ }^{33}$, les deux étant intrinsèquement liés. Ce sujet est loué pour son

24 Voy. en ce sens : Rinehart Robert and Sydor Synthia (ed.), To the Extreme. Alternative Sports, Inside Out, New York, State University of New York Press, 2003 ; Le Breton D., Conduites à risque, op. cit., pp. $176-178$; p. 230 et s.

26 Ibid., p. 26.

27 Ibid., p. 27.

28 Idem.

29 Ibid., p. 28.

30 Deleuze G., " Post-scriptum sur les sociétés de contrôle », in Pourparlers, Paris, Editions de Minuit, 1990, p. 247.

31 De Soultrait Gibus, "La question du rythme (de la vague) », in Rhuthmos, 27 décembre 2012 [en ligne], http://rhuthmos.eu/spip.php?article775 (Consulté le 27 juin 2019).

32 Rolnik S., Anthropophagie zombie, op. cit., p. 29.

33 Morizo Baptiste, "Postface", in Le loup et le musulman. Le racisme est-il une menace écologique? de Hage G., Marseille, Editions Wildproject, 2017, p. 134. 
endurance dans le changement, son dépassement de soi continuel, son habileté à s'adapter rapidement aux risques et aux vicissitudes de l'existence.

Toutefois, cette "subjectivité zombie ", appelée aussi "subjectivité-flexible-à-vendre ${ }^{34}$ par la philosophe brésilienne Suely Rolnik, loin de renouveler les manières de se rapporter au monde et à l'altérité, ne fait finalement que pousser à son paroxysme la domestication généralisée, constituant ainsi la dernière actualisation en date du sujet extractif.

L'ego occidental contemporain est cet homme complet dont parle Amos Tutuola dans L'ivrogne dans la brousse ${ }^{35}$. Un homme irrésistible en raison d'une complétude uniquement acquise par sa capacité profondément cannibale à démembrer les vivants et à s'en attribuer les organes. La « subjectivité zombie » n'est au fond qu'un crâne qui ne cesse de se construire à partir des corps des autres, qu'un trou noir autour duquel ne cesse de se reconfigurer des identités, mais qui n'existe précisément que par cette reconfiguration incessante. Point zéro de toutes ses hybridations, ce crâne est cette capacité cannibale même, intrinsèquement extractive. Et c'est précisément la confirmation de cette capacité, l'augmentation de sa puissance que recherchent certain.e.s pratiquant.e.s d'activités extrêmes.

Ainsi, l'éternelle hybridation de la "subjectivité zombie », son éternel déplacement se fait toujours à partir d'un même point fixe : elle-même. CEil du cyclone, elle demeure parfaitement stable, constante malgré les innombrables fluctuations qu' elle génère, le flot continu d'expériences et d'identités que notre société lui propose. Ce flot, loin de la remettre en question ou de la décentrer, ne fait au contraire que stimuler son appétit insatiable et pérenniser sa conception du monde selon laquelle toutes les choses existent pour être extraites, d'une façon ou d'une autre.

Le sujet occidental contemporain, aussi fluant et hybride qu'il soit, n'en demeure donc pas moins fondamentalement extractif et monoperspectiviste, incapable de concevoir une autre relation au monde que celle de la domestication

34 Rolnik S., Anthropophagie zombie, op. cit., p. 37. Cette subjectivité constitue pour Suely Rolnik la récupération et le dévoiement par le capitalisme financier de la « subjectivité flexible » qui émergea en réaction aux carcans identitaires par trop rigides du capitalisme industriel. Plongeant ses racines dans les avant-gardes intellectuelles et artistiques de la fin du XXème siècle jusqu'à la seconde guerre mondiale, cette " subjectivité flexible » était initialement profondément créatrice et inquiète, en ce qu'elle était marquée par " la capacité d'écouter les effets déstabilisateurs de l'autre en soimême » (Ibid., p. 34). Et c'est précisément ses effets déstabilisateurs qui ont été totalement éliminés lors de sa dévoration par le nouvel ordre capitaliste.

35 Tutuola Amos, L'ivrogne dans la brousse, Paris, Gallimard, 1953. Je remercie Jean-Christophe Goddard pour m'avoir permis de saisir les résonnances entre ce texte et l'anthropophagie des Tupinamba, sur laquelle repose la conception de la « subjectivité flexible » de Suely Rolnik. 
généralisée, si ce n'est sous un mode éminemment mineur. ${ }^{36}$ Son fantasme de viabilité est avant tout de se confronter au monde afin d'affirmer sa propre supériorité, son caractère exceptionnel lui permettant d'affronter le flux incessant de possibles et de défis dont le nouvel ordre capitaliste regorge. Dans une société où "l'impossible est la forme contemporaine de l'interdit "37 et donc où « tout ce qui est possible doit être expérimenté ${ }^{38}$, il s'agit de se gaver de toutes les possibilités, de les dévorer à un rythme effréné. Ce qui se retrouve ainsi valorisé, c'est tout à la fois la collection de tels exploits ${ }^{39}$ et la capacité même à les enchaîner en toute insouciance. La flexibilité et la souplesse exigées par cette nouvelle forme de subjectivité sont donc entièrement au service d'une affirmation de soi au détriment des altérités, d'une maîtrise de la nature confinée au rôle d'adversaire. ${ }^{40}$

Certain.e.s sportif.ve.s de l'extrême, en accumulant les exploits arrachés à la nature et en abordant fièrement leur insouciante flexibilité aux éléments, ne sont finalement rien d'autre qu'une horde de «zombies " parcourant le monde guidés par leur seul intérêt. Pour iels, leur environnement n'est qu'un champ d'extraction d'expériences et de sensations bénéfiques à l'accroissement de leur propre souveraineté. Dans cette optique, la vie valant la peine d'être vécue est avant toute une vie éminemment singulière, tramée d'innombrables exploits et expériences. Les éléments naturels n'y sont que des adversaires de taille leur permettant d'arborer fièrement leur qualité exceptionnelle ${ }^{41}$, leur capacité à maîtriser et à vaincre des obstacles considérés par beaucoup comme irréductibles (une falaise escarpée, une

36 « Mineur » signifie ici simplement « de moindre intensité ». En effet, pour Ghassan Hage, tout espace social est un enchevêtrement de réalités, où cohabitent différentes ontologies entretenant entre elles des rapports de force et d'intensité (Hage G., Le loup..., op. cit., p. 108). Il ne faut donc pas entendre ce terme dans le sens du concept éponyme développé par Deleuze et Guattari, bien que des rapprochements entre les deux soient évidemment possibles.

Le Breton D., Conduites à risque, op. cit., p. 81.

38 Idem.

39 Il est extrêmement révélateur que, selon le Littré, les termes « exploit » et " exploitation » aient la même origine latine (explectum), cette filiation étymologique ne faisant ainsi que confirmer leur filiation ontologique domesticatrice (Littré Emile, Dictionnaire de la langue française, tome deuxième, Paris, Librairie Hachette et Cie, 1874, p. 1571).

40 Selon cette conception, la nature est donc avant tout constituée comme une menace, mais une menace d'un genre bien particulier puisqu'elle forme également une ressource indéniable pour le développement personnel et l'affirmation de soi. Son adversité n'est recherchée qu'en tant qu'elle concourt aux intérêts de l'individu, qu'en tant qu'il peut plus ou moins la maitriser en vue de renforcer son sentiment de puissance. Purement incontrôlable, elle n'est plus qu'une menace parmi d'autres devant être exclue de notre société (Voy. en ce sens : Le Breton D., Conduites à risque, op. cit., p. 203 et s. ; Hage G., Le loup..., op. cit., p. 46 et s.).

41 Le Breton D., Conduites à risque, op. cit., p. 179. 
vague gigantesque, etc.). De la sorte, iels renforcent leur sentiment de puissance ${ }^{42}$, en estimant à appartenir à ciels sur qui même la nature n'a pas de prise.

\section{Les sports extrêmes et la diplomatie : mode d'existence mutualiste}

Pourtant, il me semble que d'autres pratiques extrêmes constituent au contraire des modalités existentielles bien différentes de la domestication généralisée, capables même d'en saper l'hégémonie actuelle. J'ai décidé de me focaliser sur l'une d'entre elles, qualifiée par Ghassan Hage de " mutualiste ${ }^{43}$, tout en reconnaissant la reconstruire quelque peu, notamment par rapport à sa conception chez Levy-Bruhl. Ce choix s'explique non seulement parce que cette conception du monde me semble particulièrement pertinente au regard des enjeux écologiques contemporains, mais également parce qu' elle est omniprésente chez un certain nombre de pratiquant.e.s de l'extrême.

\subsection{Le mutualisme - Exister les un.e.s dans les autres}

Ce mutualisme peut être rapproché de ce que d'aucun.e.s nomment « l'ecopsychology ", courant tant théorique que pratique mettant en avant l'appartenance de l'être humain à la nature, la profonde connexion existante entre nous et le monde. ${ }^{44}$ Selon ce courant, nous ne pouvons nous comprendre nous-mêmes qu'en tant que partie prenante de la nature. Dans ce type de relation constitutive, "nous nous sentons et nous sentons les autres 'participer' à la vie de chacun d'entre nous $»^{45}$. Le monde n'est plus un ensemble d'outils, un espace habitable organisé exclusivement autour de nos intérêts personnels ${ }^{46}$, mais bien plutôt un mouvement avec lequel nous nous sentons en communion, auquel nous participons et qui participe en nous.

En raison du fait que nous existons les un.e.s dans les autres, que nos existences sont étroitement intriquées, l'augmentation de la force vitale de l'un.e se propage nécessairement à l'autre. ${ }^{47}$ À l'inverse de la domestication généralisée, où l'augmentation de la souveraineté de l'autre était vécue comme un affaiblissement

\footnotetext{
Ibid., p. 174.

Hage H., Le loup..., op. cit., p. 101.

Brymer E., Downey Greg, Gray T., « Extreme Sports as Precursor to Environmental Substainability ", in Journal of Sport and Tourism, 14/2-3, May-August 2009, p. 197.

Hage G., Le loup..., op. cit., p. 102.

Ibid., p. 86.

Ibid., p. 103.
} 
de ma propre souveraineté, ici, nous nous sentons participer à la même énergie, à la même puissance. Ainsi, dans cette modalité d'existence, il nous faut bien plus nous insérer dans un mouvement que le produire ${ }^{48}$, négocier avec lui plutôt que le maîtriser.

\subsection{Sports extrêmes - L'art subtil de la négociation avec la nature}

Or, ce fantasme de viabilité se retrouve chez de nombreux sportif.ve.s de l'extrême, et tout particulièrement chez les vétérant.e. $s^{49}$. En effet, bon nombre d'entre iels éprouvent leur pratique avant tout comme « une danse avec la nature ${ }^{50}$ où iels fusionnent avec les éléments en entrant en négociations avec eux. ${ }^{51}$ Par exemple Lynn Hill, grimpeuse professionnelle, explique que, pour elle, escalader " [is] not about going out and conquering something-proving that you are somehow stronger than other people or the rock you're about to climb. It is much more about interacting with your environment. $»^{52}$

D'autres mettent l'accent sur cette idée de partage d'une même énergie, à mille lieues de la logique extractive selon laquelle nous devons nécessairement affaiblir autrui pour augmenter notre propre puissance..$^{53}$ Ainsi, un surfeur d'une cinquantaine d'années raconte la manière dont il se sent connecté à l'énergie d'une plage de surf, la façon dont celle-ci se propage à travers lui : "You get great energy, fantastic energy on the sort of coast where there's big surf; you feel the energy, it's just amazing... You know, there's a lot of energy, and you get off on that energy. » ${ }^{54}$

Toutefois, cette interaction nécessite une connaissance, une négociation permanente avec le monde et ses différentes composantes. Bien loin de l'image d'Épinal que certaines conceptions anthropologiques véhiculent, ce mutualisme nécessite une vigilance constante, un art subtil de la diplomatie avec la nature et les différentes formes de vie. ${ }^{55}$ Yves Bessas, pionnier français des sports extrêmes, déclare ainsi que : « Les libéristes ${ }^{56}$ qui se sont écrasés contre des falaises ou sont tombés en chute libre ou ont été emmenés par un courant ascendant ne se comptent

\footnotetext{
De Soultrait G., « La question du rythme (de la vague) », art. cit.

Brymer E., Downey G., Gray T., « Extreme Sports as Precursor... », art. cit., p. 195.

Brymer E., Gray T., "Dancing with Nature...», art. cit., p. 136.

Le Breton D., Conduites à risque, op. cit., p. 259.

Brymer E., Gray T., « Dancing with Nature... », art. cit., p. 142. C'est moi qui souligne.

Hage G., Le loup..., op. cit., p. 51.

Brymer E., Downey G., Gray T., « Extreme Sports as Precursor... », art. cit., p. 201.

Morizot Baptiste, « Postface », in Le loup..., op. cit., p. 136.

Pratiquant.e.s du vol à la voile avec une aile libre.
} 
plus. Ils n'ont pas su apprendre à respecter ce que la nature leur enseignait ${ }^{57}$. Laurence de la Ferrière, première Française à avoir atteint le pôle Sud sans assistance, explique quant à elle que là-bas « on est dans un autre monde, il faut l'apprivoiser, muter, se faire adopter. Cela nécessite une très grande humilité. " ${ }^{58}$

Loin de considérer « qu' on est chez soi que seul » 59 , et que l'on peut habiter un espace en toute ignorance des éléments qui nous entourent, cette modalité existentielle postule au contraire qu'il nous faut nécessairement partager notre habitat et être attentif.ve.s, dans tous les sens du terme, à l'ensemble des entités qui y participent. Comme le précise bien Baptiste Morizot, «c'est un mode d'habiter dans un monde simultanément plus aventureux et plus hospitalier. ${ }^{60}$

Dans cette optique, bien loin de renforcer et de légitimer leur toute-puissance extractive, ces pratiques invitent au contraire les sportif.ve.s extrêmes à la modestie en leur faisant prendre conscience de leur vulnérabilité et de leur insignifiance au sein du monde. Par exemple, une alpiniste déclare : "I would say that in a wild environment, even if you don't open your heart to it, you are vulnerable because that's just the way it is. You are just a tiny little thing, very fragile... It's about feeling at one with the whole world, the whole universe. ${ }^{61}$

Ainsi, certain.e.s d'entre iels semblent avoir vu leur relation à la nature profondément évoluée grâce à leur pratique. Pour iels, il n’est plus question de la dominer, d'y affirmer leur souveraineté en maitrisant et écartant les obstacles qu'elle place sur leur route, mais bien d'interagir avec elle, de négocier avec ses éléments. Eric Brymer et Tonia Gray ont démontré dans leur étude que la pratique de telles activités permet au contraire aux sportif.ve.s de diminuer leur besoin de contrôle et de domination de la nature, en leur faisant prendre conscience qu'iels y sont inextricablement lié.e.s, et inversement. ${ }^{62} \mathrm{Ce}$ genre de pratiques peut donc servir de puissant catalyseur d'une véritable prise de conscience écologique ${ }^{63}$, qui ne se résumerait pas à défendre l'environnement exclusivement en vue de nos propres intérêts, tels que la préservation de notre spot préféré ou la pérennité de notre activité économique. ${ }^{64}$

\footnotetext{
Bessas Yves, La glisse, Paris, Fayard, 1982, p. 174. Cité par Le Breton D., Conduites à risque, op. cit., p. 259.

Le Breton D., Conduites à risque, op. cit., p. 260.

9 Morizot B., «Postface» in Le loup..., op. cit., p. 134.

Ibid., p. 136.

Brymer E., Downey G., Gray T., « Extreme Sports as Precursor... », art. cit., p. 200.

Brymer E., Gray T., « Dancing with Nature... ", art. cit., pp. 135-149.

Ibid., p. 145.

Brymer E., Downey G., Gray T., « Extreme Sports as Precursor... », art. cit., p. 195.
} 


\section{Conclusion}

En guise de conclusion, j'aimerais quelque peu revenir sur mes expériences boliviennes, afin de m'interroger sur le ou les fantasmes de viabilité qui les habitaient. Il me semble évident qu'à première vue, celles-ci furent l'œuvre d'un «zombie cannibale » démembrant des espaces dont il ne connaissait rien pour les arborer fièrement et accroître ainsi son sentiment de puissance. Il s'agissait avant tout de vaincre Huayna Potosi et le Camino de la muerte, de les conquérir en considérant les éléments naturels, tels que la glace, le vent ou les falaises, comme des obstacles à ma souveraineté, des contraintes à écarter. Le décuplement de ma puissance passait nécessairement par l'affaiblissement et la maîtrise de mon environnement.

Mais, me semble-t-il, une autre modalité existentielle, davantage mutualiste, était déjà présente dans ces exploits, bien que sur un mode éminemment mineur. En effet, en réalisant ces différentes expériences en compagnie de guides chevronnés, j'avais peut-être déjà conscience de la nécessaire diplomatie que ma présence en ces lieux requérait. Je reconnaissais qu'il me fallait des traducteurs pour communiquer avec le milieu, négocier finement avec lui plutôt que le conquérir brutalement. Mais l'existence de ce mode de relation spécifique n'était qu'anecdotique sur ma " carte ontologique ${ }^{65}$, et fit à peine trembler les fondations solidement ancrées du monopole de la domestication généralisée.

Or l'urgence climatique exige de nous une écologie radicale, c'est-à-dire une écologie qui remonte jusqu'à la racine du problème, retrace nos cartes ontologiques et brise l'hégémonie de la domestication généralisée imposée au monde entier par l'Occident. L'enjeu majeur de cette crise écologique n'est donc pas de colmater les brèches, de simplement limiter les excès de cette modalité existentielle, mais bien de la remettre fondamentalement en question en faisant la part belle à d'autres manières de concevoir et d'habiter le monde. Il nous faut modifier en profondeur nos fantasmes de viabilité, nos manières d'être, afin de constituer, par exemple, un monde auquel nous participerions pleinement et avec lequel nous devrions nécessairement apprendre à négocier.

À cet égard, une certaine manière de pratiquer les sports extrêmes, bien plus axée sur la collaboration et l'interaction que sur la domination et la conquête, constitue une piste particulièrement féconde, notamment d'un point de vue éducationnel. Ces pratiques créent en effet un monde nouveau qui n'est plus organisé exclusivement en fonction de l'intérêt personnel et des valeurs que nous pouvons

65 Morizot B., «Postface », in Le loup..., op. cit., p. 134. 
en retirer. Elles nous apprennent au contraire à négocier avec la nature, à nous couler dans son rythme plutôt qu'à lui imposer le nôtre, et participent ainsi à cette écologie radicale pour laquelle il n'est plus tant question de changer le monde que de changer de mondes.

Gauthier Dierickx est titulaire d'un Master en droit de l'Université libre de Bruxelles et d'un Bachelier en philosophie de l'Université Saint-Louis-Bruxelles, il poursuit actuellement ses études en philosophie dans le cadre du Master Conjoint Erasmus Mundus «Philosophies allemandes et françaises : enjeux contemporains » (PhiAFEC). Il s'intéresse plus particulièrement aux questions de traductions ontologiques et à la relation entre désirs et institutions, à l'entrecroisement du droit, de la philosophie et de l'anthropologie.

E-mail : gauthier.dierickx@icloud.com 